\title{
Finite Difference Implicit Schemes to Coupled Two-Dimension Reaction Diffusion System
}

\author{
Shahid Hasnain', Muhammad Saqib ${ }^{2}$, Nawaf Al-Harbi ${ }^{1}$ \\ ${ }^{1}$ Department of Mathematics, Numerical Analysis Division, King Abdulaziz University, Jeddah, KSA \\ ${ }^{2}$ Department of Mathematics, Air University, Islamabad, Pakistan \\ Email: shahidqa32@gmail.com, saqibmurree@gmail.com,nawaf0558@hotmail.com
}

How to cite this paper: Hasnain, S., Saqib, M. and Al-Harbi, N. (2018) Finite Difference Implicit Schemes to Coupled Two-Dimension Reaction Diffusion System. Journal of Applied Mathematics and Physics, 6, 737-753. https://doi.org/10.4236/jamp.2018.64066

Received: January 26, 2018

Accepted: April 17, 2018

Published: April 20, 2018

Copyright ( 92018 by authors and Scientific Research Publishing Inc. This work is licensed under the Creative Commons Attribution International License (CC BY 4.0).

http://creativecommons.org/licenses/by/4.0/

\begin{abstract}
In this research article, two finite difference implicit numerical schemes are described to approximate the numerical solution of the two-dimension modified reaction diffusion Fisher's system which exists in coupled form. Finite difference implicit schemes show unconditionally stable and second-order accurate nature of computational algorithm also the validation and comparison of analytical solution, are done through the examples having known analytical solution. It is found that the numerical schemes are in excellent agreement with the analytical solution. We found, second-implicit scheme is much faster than the first with good rate of convergence also we used NVIDA devices to accelerate the computations and efficiency of the algorithm. Numerical results show our proposed schemes with use of HPC (High performance computing) are very efficient and reliable.
\end{abstract}

\section{Keywords}

Crank Nicolson, Taylor's Series, Richardson Extrapolation and Alternating Direction Implicit

\section{Introduction}

Reaction diffusion (RD) equations rise up naturally in systems consisting of many interacting factors, such as chemical reactions and are widely used to identify pattern formation phenomena in diverseness of biological and physical systems [1]. The primary ingredients of all these models are in the form of mathematical balance equation [1] which is in two dimensions

$$
\partial_{t} u=\beta\left(\nabla^{2} u\right)+\boldsymbol{R}(u) \text {, }
$$

where $u=u(x, y, t)$ is a vector of concentration variables, $R(u)$ describes a local 
reaction kinetics and the Laplace operator $\nabla^{2}$ acts on the vector $u$ component wise, also $\boldsymbol{\beta}$ denotes a diagonal diffusion coefficient matrix [1]. Noted that we suppose the system to be isotropic and uniform, so $\beta$ is represented by a scalar matrix, independent on coordinates of the system [1]. Research in this field starts form the classical papers of [1] [2] [3], incited by population dynamics issues, where researchers made modified diffusion equation:

$$
\partial_{t} u(x, y, t)=\boldsymbol{\beta}\left(u_{x x}+u_{y y}\right)+\boldsymbol{R}(u)
$$

with a nonlinear source term $R(u)=u-u^{2}$ [2] [3] [4]. A typical solution of the Equation (1), a propagating front, separating two non-equilibrium homogeneous states, one of which $(u=1)$ is stable and another one $(u=0)$ is unstable, such fronts behavior is often said to be propagation into stable state and also other referred to as waves (or fronts) of transition from an unstable state [3] [4] [5]. The interest in these fronts was stimulated in the early 1980s, such as these fronts can be found in various physical, chemical as well as biological systems [5] [6].

The reaction diffusion Equation (2) represents a model equation for the evolution of a neutron population in a nuclear reactor and also arises in chemical engineering applications, such equation allows for the effects of linear diffusion by means of $\boldsymbol{u}_{x x}+\boldsymbol{u}_{y y}$ and nonlinear local multiplication or reaction through $\boldsymbol{R}(u)$ [7] [8].

Researchers have studied these model problems such as the stability of symmetric traveling waves in the Cauchy problem for a more general case than Equation (2); also some researchers explained perturbation method and found an approximate solution by expanding the solution in terms of a power series and in terms of some small parameters [8].

In this paper, we suggest a reaction diffusion system, which agree to several physical phenomena, the most common is the change in space and time of the concentration of one or more chemical substances. Local chemical reactions in which the metamorphosed into each other, and diffusion which causes the substances to spread out over a surface in space. Reaction diffusion systems are naturally applied in chemistry. However, the system can also describe dynamical processes of non-chemical nature. Mathematically, reaction diffusion systems take the form of semilinear parabolic partial differential equations. The general form of our proposed reaction diffusion system in two dimension, is

$$
\begin{gathered}
u_{t}=\boldsymbol{\beta}\left(u_{x x}+u_{y y}\right)+u^{2} v-\alpha u, \quad(x, y) \in(-\infty, \infty), t \geq 0 \\
v_{t}=\boldsymbol{\beta}\left(v_{x x}+v_{y y}\right)-v^{2} u, \quad(x, y) \in(-\infty, \infty), t \geq 0
\end{gathered}
$$

where $\beta$ is diffusion coefficient and $\alpha$ is a reactive factor, and $u(x, t)$ is concentration and $v(x, t)$ is the velocity of the chemical reaction. The aim of this work is to look into the viability of finite difference schemes for the numerical solution of two-dimension coupled reaction diffusion system. The proposed finite difference schemes show good agreement with analytical solution along 
efficiency in time. Comparison of two finite difference (FD) schemes is also mentioned with CPU efficiency.

The outlook of the paper is in Section 2 analytical solution, Section 3 smoothness and uniqueness, Section 4 numerical methods, Section 5 numerical results and Section 6 discussion.

\section{Analytical Solution}

To derive the analytical solution of the given system in (3), (4), we assume the solution of the two dimension coupled reaction diffusion system, in the following form

$$
\begin{gathered}
u(x, y, t)=\mathrm{e}^{\alpha \frac{t}{2}-x-y} \\
v(x, y, t)=\mathrm{e}^{-\alpha \frac{t}{2}+x+y}
\end{gathered}
$$

\section{Smoothness and Uniqueness of the Reaction Diffusion System}

In order to guarantee the smoothness and uniqueness of a positive solution and to obtain upper and lower bounds of the solution, it is necessary to impose some general assumptions on the various physical parameters and the reaction function [9] [10]. Throughout this paper, we always assume that the diffusion coefficient $\beta$ is positive in domain $\Omega$ also at $t=0$ the initial values $u_{0}, v_{0}$ are non-negative, where the smoothness hypothesis is used only for the existence problem of the corresponding linear system, and the non-negative hypothesis on the data is to obtain non-negative solutions [9] [10]. Let us consider the system,

$$
\begin{aligned}
& \left.\begin{array}{l}
u_{t}-\nabla \cdot(\beta \nabla u)=-u^{m} f(u, v) \\
v_{t}-\nabla \cdot(\beta \nabla v)=u^{m} g(u, v)
\end{array}\right\} \\
& \text { where } m \geq 1, f(u, v)=u v-\alpha \text { and } g(u, v)=v^{2} \\
& \left.\begin{array}{l}
B_{1}[u]=h_{1}(x, y) \\
B_{2}[v]=h_{2}(x, y)
\end{array}\right\} \\
& \text { Boundary Conditions, } t>0,(x, y) \in \Omega \\
& \left.\begin{array}{l}
u(0, x, y)=u_{0}(x, y) \\
v(0, x, y)=v_{0}(x, y)
\end{array}\right\} \\
& \text { Initial Conditions, }(x, y) \in \Omega
\end{aligned}
$$

motivated by the nonlinear reaction functions given by Equation (7), we make the following basic assumption on functions $f$ and $g$ [9] [10].

\subsection{Assumption or Hypothesis $(\mathrm{H})$}

$\partial f / \partial v$ exists and is bounded subsets of domain $\Omega$ and there exists a function with $c_{o}(x, y) \geq 0$, such that $0 \leq f\left(u, v_{1}\right) \leq f\left(u, v_{2}\right) \leq c_{o}(x, y)$ for $0 \leq v_{1} \leq v_{2} \leq \infty$ [9] [10]. This definition implies that the function $f$ is monotone non-decreasing in $V$ and is uniform bounded for $v \geq 0$ [9] [10]. Clearly this condition is satisfied by 
both functions $f$ and $g$, thus this property leads to

$$
\left.\begin{array}{l}
F_{1}(x, y, u, v)=-u^{m} f(u, v) \\
F_{2}(x, y, u, v)=+u^{m} g(u, v),
\end{array}\right\}
$$

where above Equation (8) represents $F_{1}, F_{2}$ are quasi monotone non-increasing and quasi monotone non-decreasing functions in $\Omega$ respectively [9] [10]. According to classification of the reaction functions, $F_{1}, F_{2}$ are typed III functions [9] [10], which leads to the following definition of the solutions.

\subsection{Definition}

A smooth pair of two vector functions $(\breve{u}, \breve{v}),(\bar{u}, \bar{v})$ defined in $\mathbf{R}^{+} \times \Omega$ are called upper and lower solutions respectively, if they satisfy the following inequalities

$$
\begin{aligned}
& \breve{u}_{t}-\nabla \cdot(\beta \nabla \breve{u})+\breve{u}^{m} f(x, \bar{v}) \geq 0 \geq \bar{u}_{t}-\nabla \cdot(\beta \nabla \bar{u})+\bar{u}^{m} f(x, y, \breve{v}) \\
& \left.\breve{v}_{t}-\nabla \cdot(\beta \nabla \breve{v})-\breve{u}^{m} g(x, \breve{v}) \geq 0 \geq \bar{v}_{t}-\nabla \cdot(\beta \nabla \bar{v})-\breve{u}^{m} g(x, y, \bar{v})\right\} \\
& \text { where } m \geq 1, f(x, y, v)=u \breve{v}-\alpha \text { and } g(x, y, v)=\bar{v}^{2} \\
& B_{1}[\breve{u}] \geq h_{1}(x, y) \geq B_{1}[\bar{u}] \\
& \left.B_{2}[\bar{v}] \geq h_{2}(x, y) \geq B_{2}[\bar{u}]\right\} \\
& \left.\breve{u}(0, x, y) \geq u_{0}(x, y) \geq \bar{u}(0, x, y)\right) \\
& \left.\breve{v}(0, x, y) \geq v_{0}(x, y) \geq \bar{v}(0, x, y)\right\} \\
& \text { Initial Conditions, }(x, y) \in \Omega
\end{aligned}
$$

In the above definitions the smoothness of $(\breve{u}, \bar{v}),(\bar{u}, \bar{v})$ is in the sense that these functions are continuously differentiable to the order appeared in Equations (7) and (8) respectively [9] [10]. Hypothesis and above definition leads to the following theorem.

\subsection{Theorem}

Let $\mathrm{f}$ and $\mathrm{g}$ satisfy above hypothesis $(\mathrm{H})$. If there exist upper and lower solutions $(\breve{u}, \breve{v}),(\bar{u}, \bar{v})$ of system 7 , such that $\breve{u} \leq \bar{u}$ and $\breve{v} \leq \bar{v}$ in $\mathbf{R}^{+} \times \Omega$, then the sequence $\left\{\dot{u}^{k}, \dot{v}^{k}\right\},\left\{\underline{u}^{k}, \underline{v}^{k}\right\}$ converges monotonically from above and below, respectively, to a unique solution $(u, v)$ of system (7) [9] [10]. Moreover

$$
\left.\begin{array}{l}
\breve{u}(t, x, y) \leq u(t, x, y) \leq \bar{u}(t, x, y) \\
\breve{v}(t, x, y) \leq v(t, x, y) \leq \bar{v}(t, x, y)
\end{array}\right\} t>0,(x, y) \in \Omega
$$

The usefulness of the above theorem is that through suitable construction of upper and lower solutions, not only can the existence problem be ensured, but the stability and the asymptotic behavior of the time dependent solution can also be established from the behavior of the upper and lower solutions [9] [10]. Thus, the definition of stability of a steady state solution is in the usual sense of Lyapunov function [9] [10]. Unlike scalar systems or coupled systems with qua- 
si-monotone increasing function, the upper and lower solutions for the present system are interconnected and to be determined simultaneously from relations in Equation (9). This makes the determination of these functions more delicate, especially in relation to the stability property of non-homogeneous systems [9] [10]. Nevertheless, for the global existence problem or the stability problem with homogeneous boundary conditions the construction of those functions is not very difficult [10].

\section{Numerical Methods}

We consider the numerical solution of the nonlinear system in (5), (6) and (7) in a finite domain $\Omega=\{(x, y) \mid a<x<b, c<y<d\}$, where the first step is to choose integers $n$ and $\mathrm{m}$ to define step sizes $h=(b-a) / n$ and $k=(d-c) / m$ [11] [12]. Partition the interval [a, b] into $\mathrm{n}$ equal parts of width $\mathrm{h}$ and the interval [c, d] into m equal parts of width $k$ and place a grid on the rectangle $R$ by drawing vertical and horizontal lines through the points with coordinates $\left(x_{i}, y_{j}\right)$, where $x_{i}=a+i h$ for each $i=0,1,2, \cdots, n$ and $y_{j}=c+j k$ for each $j=0,1,2, \cdots, m$ also the lines $x=x_{i}$ and $y=y_{j}$ are grid lines, and their intersections are the mesh points of the grid [12] [13] [14] [15] [16]. For each mesh point in the interior of the grid, $\left(x_{i}, y_{j}\right)$, for $i=1,2, \cdots, n-1$ and $j=1,2, \cdots, m-1$, also we assume $t_{n}=n t, n=0,1, \cdots$ where $t$ is the time grid step size [16]. We denote the analytical and numerical solutions at the grid point $\left(x_{m}, t_{n}\right)$ by $u_{m}^{n}$ and $U_{m}^{n}$ respectively.

\subsection{Second Order Implicit Scheme}

The Crank Nicolson scheme for the system in (3) and (4) can be displayed as follows:

$$
\left.\begin{array}{l}
\hat{u}=\frac{u_{l, m}^{n+1}+u_{l, m}^{n}}{2} \\
\hat{v}=\frac{v_{l, m}^{n+1}+v_{l, m}^{n}}{2} \\
\delta_{x}^{2} \hat{u}=\frac{\hat{u}_{l+1, m}-2 \hat{u}_{l, m}+\hat{u}_{l-1, m}}{h^{2}} \\
\delta_{y}^{2} \hat{u}=\frac{\hat{u}_{l, m+1}-2 \hat{u}_{l, m}+\hat{u}_{l, m-1}}{h^{2}} \\
\delta_{x}^{2} \hat{v}=\frac{\hat{v}_{l+1, m}-2 \hat{v}_{l, m}+\hat{v}_{l-1, m}}{h^{2}} \\
\delta_{y}^{2} \hat{v}=\frac{\hat{v}_{l, m+1}-2 \hat{v}_{l, m}+\hat{v}_{l, m-1}}{h^{2}} \\
u_{l, m}^{n+1}-u_{l, m}^{n}-\mathbf{R}_{1}\left(\delta_{x}^{2}+\delta_{y}^{2}\right) \hat{u}-\frac{k}{8}\left(\hat{v} \hat{u}^{2}\right)+0.5 k(\hat{u})=0 \\
v_{l, m}^{n+1}-v_{l, m}^{n}-\mathbf{R}_{1}\left(\delta_{x}^{2}+\delta_{y}^{2}\right) \hat{v}+\frac{k}{8}\left(\hat{v}^{2} \hat{u}\right)=0,
\end{array}\right\}
$$

where $\mathbf{R}_{1}=\frac{k \beta}{h^{2}}$ [17] [18]. The scheme in Equation (11) is a nonlinear implicit 
scheme with block linear penta diagonal structure [17] [18]. The Newton's iterative method is used to solve this linear system, such scheme is of second order accuracy in both directions, space and time respectively [17] [18]. The scheme is unconditionally stable using the von Neumann stability analysis [18].

\subsection{Computationally Efficient Implicit Scheme}

In search of a time efficient alternate, we analyzed the naive version of the Crank Nicolson scheme for the two dimensional equation, and find out that that scheme is not time efficient such that to get time efficiency, the common name of Alternating Direction Implicit (ADI) method can be used [19] [20]. In ADI scheme, the two steps are as follows:

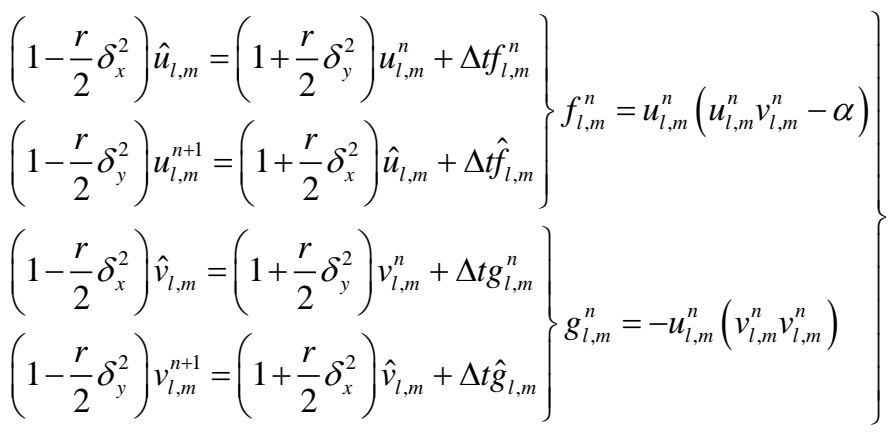

The trick used in constructing of ADI scheme, is to split time step into two sweeps and apply two different stencils in each half time step, therefore to increment time by one time step in grid point, we first compute both of these stencils, such that the resulting linear system is block tridiagonal [20] [21] [22]. The scheme in Equation (12) is a nonlinear implicit scheme of second order accuracy in space and time. According to Von Neumann stability analysis, such scheme is also unconditionally stable [22] [23] [24] [25].

\subsection{Algorithm}

The nonlinear system of Equation (12), can be written in the form:

$$
R(W)=0,
$$

where $R=\left(r_{1}, r_{2}, r_{3}, \cdots, r_{2 n}\right)^{t}, W=\left(u_{1}^{n+1}, v_{1}^{n+1}, u_{2}^{n+1}, v_{2}^{n+1}, \cdots, u_{m}^{n+1}, v_{m}^{n+1}\right)$ and $r_{1}, r_{2}, r_{3}, \cdots, r_{2 n}$ are the nonlinear equations obtained from the system in Equation (12). The system of Equations in (12) is solved by Newton's iterative method using the following steps:

1) Specify $W^{(0)}$ as an initial approximation.

2) For $k=0,1,2, \cdots$ until convergence achieve.

- Solve the linear system $A\left(W^{(k)}\right) \Delta W^{(k)}=-R\left(W^{(k)}\right)$

- Specify $W^{(k+1)}=W^{(k)}+\Delta W^{(k)}$,

where $A\left(W^{(k)}\right)$ is $(m \times m)$ Jacobian matrix, which is computed analytically and $\Delta W^{(k)}$ is the correction vector [26] [27]. In the iteration method solution at the previous time step is taken as the initial guess. Iteration at each time step is 
stopped when $\left\|R\left(W^{(k)}\right)\right\|_{\infty} \leq$ Tol with Tol is a very small prescribed value. The linear system obtained from Newton's iterative method, is solved by Gauss elimination method with partial pivoting also convergence done with iterations along less CPU time [28] [29] [30].

\subsection{Norms}

The accuracy and consistency of the schemes is measured in terms of error norms specially $L_{2}$ and $L_{\infty}$ which are defined as:

$$
\left.\begin{array}{l}
L_{\infty}=\left\|u^{\text {ecact }}-u^{\text {Approximation }}\right\|_{\infty}=\max _{1 \leq i \leq m} \sum_{j=1}^{m}\left|u_{j}^{\text {ecact }}-u_{j}^{\text {Approximation }}\right| \\
L_{2}=\left\|u^{\text {ecact }}-u^{\text {Approximation }}\right\|_{2}=\sqrt{\sum_{j=1}^{m}\left|u_{j}^{\text {ecact }}-u_{j}^{\text {Approximation }}\right|} \\
\text { Rate }=\frac{\log \left(\text { Error }_{h} / \text { Error }_{h / 2}\right)}{\log (h /(h / 2))}
\end{array}\right\}
$$

Two more interesting error are listed below,

$$
\left.\begin{array}{l}
\text { Error }_{\text {relative }}=\sqrt{\frac{\sum_{i} \sum_{j}\left|u_{i, j}^{\text {ecact }}-u_{i, j}^{\text {Approximation }}\right|^{2}}{\sum_{j}\left|u_{i, j}^{\text {exact }}\right|^{2}}} \\
\text { RMS }=\sqrt{\frac{\sum_{i} \sum_{j}\left(u_{i, j}^{\text {ecact }}-u_{i, j}^{\text {Approximation }}\right)^{2}}{M^{2}}}, \text { where } M=\text { Total terms }
\end{array}\right\}
$$

\section{Numerical Results}

Numerical computations have been performed using the uniform grid, for the test problem, the approximated and analytical solutions such as $u(x, y, t)$ and $U(x, y, t)$ have been given in Table 1 , Table 2 at different grids with some fixed parameters such as $\alpha=1, \beta=1 / 4$ and $k=0.0001$ and $t=1$ using implicit Crank Nicolson scheme. Graphically, Figures 1-2 explain Crank Nicolson scheme with different parameters while Figures 3-4 indicate ADI scheme with error profile in Figures 5-6. Also in Tables 3-8, we analyzed some more feathers using ADI scheme at different grids along the same parameters as we mentioned above. We discussed some interesting feathers regarding computer system using ADI scheme in Table 9, Table 10, along Self time which is the time spent in a function excluding the time spent in its child functions [29] [30] [31] [32]. Self time, includes overhead resulting from the process of profiling, such as Child functions are involved in coding of such problems [29] [30] [31] [32]. Thus later scheme works very fine for half spacing as we improve accuracy, which is lead by Richardon extrapolation method, see from Table 10. 
Table 1. Error comparison by crank nicolson scheme at different grid sizes, at $t=1$ and time step $=k=0.0001$.

\begin{tabular}{ccccc}
\hline Grid Size & Error $_{\text {relative }}$ & RMS & $L_{2}$ & $L_{\infty}$ \\
\hline $11 \times 11$ & 0.0089 & 0.0152 & 0.1671 & 0.0285 \\
$21 \times 21$ & 0.0091 & 0.0312 & 0.1869 & 0.0463 \\
$31 \times 31$ & 0.0091 & 0.0403 & 0.2139 & 0.0512 \\
$41 \times 41$ & 0.0095 & 0.0679 & 0.3913 & 0.0710 \\
$51 \times 51$ & 0.0099 & 0.0931 & 0.5524 & 0.0989 \\
\hline
\end{tabular}

Table 2. Rate of convergence comparison by crank nicolson scheme at different grid sizes at $t=1$ and time step $=k=0.0001$.

\begin{tabular}{ccccc}
\hline Grid Size & Rate $_{L_{2}}$ & Rate $_{L_{\infty}}$ & $L_{2}$ & $L_{\infty}$ \\
\hline $11 \times 11$ & 2.9385 & 2.0235 & 0.1671 & 0.0285 \\
$21 \times 21$ & 2.2367 & 1.9896 & 0.1869 & 0.0463 \\
$31 \times 31$ & 1.8976 & 1.4781 & 0.2139 & 0.0512 \\
$41 \times 41$ & 1.2145 & 1.2797 & 0.3913 & 0.0710 \\
$51 \times 51$ & 1.1135 & 1.0923 & 0.5524 & 0.0989 \\
\hline
\end{tabular}

Table 3. Solution by ADI scheme at different locations at $t=1$, time step $=k=0.0001$ and grid size $=25 \times 25$.

\begin{tabular}{cccc}
\hline Space Location & $u_{\text {app. }}$ & $U_{\text {Analyical }}$ & Error $_{u-U}$ \\
\hline$(0.0152,0.1667)$ & 0.494155003687347 & 0.494367466355141 & 0.00021246 \\
$(0.0455,0.1970)$ & 0.53835177275621 & 0.535255683530614 & 0.0031 \\
$(0.0606,0.2424)$ & 0.565563567758470 & 0.558073805129842 & 0.0075 \\
$(0.1970,0.1970)$ & 0.626597867998406 & 0.621185376572772 & 0.0054 \\
$(0.2879,0.2879)$ & 0.749945814748917 & 0.739953358282411 & 0.0100 \\
\hline
\end{tabular}

Table 4. Error comparison by ADI scheme at different grid sizes, at $t=1$ and time step $=$ $k=0.0001$.

\begin{tabular}{ccccc}
\hline Grid Size & Error $_{\text {reative }}$ & RMS & $L_{2}$ & $L_{\infty}$ \\
\hline $11 \times 11$ & 0.0741 & 0.0173 & 0.1899 & 0.0713 \\
$21 \times 21$ & 0.0341 & 0.0283 & 0.5934 & 0.0428 \\
$31 \times 31$ & 0.0123 & 0.0362 & 1.1231 & 0.0232 \\
$41 \times 41$ & 0.0099 & 0.0428 & 1.7538 & 0.0155 \\
$51 \times 51$ & 0.0090 & 0.0485 & 2.4713 & 0.0136 \\
\hline
\end{tabular}

Table 5. Error comparison by ADI scheme at different time with time steps $=k=0.0001$.

\begin{tabular}{ccccc}
\hline$t=$ time & Error $_{\text {relative }}$ & RMS & $L_{2}$ & $L_{\infty}$ \\
\hline 0.01 & 0.00013 & 0.0102 & 1.04713 & 0.00198 \\
0.05 & 0.00089 & 0.0209 & 1.09513 & 0.00589 \\
0.1 & 0.0011 & 0.0319 & 1.12686 & 0.00989 \\
0.5 & 0.0039 & 0.0400 & 1.8535 & 0.0109 \\
1 & 0.0090 & 0.0485 & 2.4713 & 0.0136 \\
\hline
\end{tabular}


Table 6. Error comparison by ADI scheme at different time steps.

\begin{tabular}{ccccc}
\hline$k=$ time steps & Error $_{\text {relative }}$ & RMS & $L_{2}$ & $L_{\infty}$ \\
\hline 0.01 & 0.0484 & 0.2942 & 2.3542 & 0.1236 \\
0.001 & 0.0483 & 0.2936 & 2.3393 & 0.1240 \\
0.0001 & 0.0461 & 0.2935 & 2.3380 & 0.1241 \\
0.00001 & 0.0423 & 0.2935 & 2.3378 & 0.1241 \\
\hline
\end{tabular}

Table 7. Rate of Convergence Comparison by ADI scheme at different grid sizes.

\begin{tabular}{ccccc}
\hline Grid Size & Rate $_{L_{2}}$ & Rate $_{L_{\infty}}$ & $L_{2}$ & $L_{\infty}$ \\
\hline $11 \times 11$ & 2.8009 & 2.8201 & 0.1899 & 0.0113 \\
$21 \times 21$ & 2.8458 & 2.8733 & 0.5934 & 0.0128 \\
$31 \times 31$ & 2.8619 & 2.8934 & 1.1231 & 0.0132 \\
$51 \times 51$ & 2.8458 & 2.8733 & 2.4713 & 0.0136 \\
\hline
\end{tabular}

Table 8. Error Comparison by ADI scheme at different space step sizes.

\begin{tabular}{ccccc}
\hline$h$ & Error $_{\text {relative }}$ & RMS & $L_{2}$ & $L_{\infty}$ \\
\hline 0.04 & 0.0483 & 0.2936 & 7.3393 & 0.1240 \\
0.02 & 0.0118 & 0.0406 & 1.0159 & 0.0168 \\
0.01 & 0.0107 & 0.0357 & 0.8920 & 0.0148 \\
0.005 & 0.0072 & 0.0219 & 0.5484 & 0.0090 \\
0.000625 & 0.0002 & 0.0118 & 0.0253 & 0.0011 \\
\hline
\end{tabular}

Table 9. Interesting feathers of ADI scheme at different grid sizes.

\begin{tabular}{ccccc}
\hline Grid Size & Self Time & Total Time & CTC. Function & Convergence Rate \\
\hline $11 \times 11$ & $0.312 \mathrm{~s}$ & $0.743 \mathrm{~s}$ & 12,642 & 2.8201 \\
$31 \times 31$ & $1.094 \mathrm{~s}$ & $2.291 \mathrm{~s}$ & 38,322 & 2.8934 \\
$51 \times 51$ & $1.968 \mathrm{~s}$ & $4.030 \mathrm{~s}$ & 65,602 & 2.8733 \\
\hline
\end{tabular}

Table 10. Interesting feathers of ADI scheme at different grid sizes.

\begin{tabular}{ccccc}
\hline Grid Spacing & Self Time & Total Time & CTC. Function & Convergence Rate \\
\hline$h$ & $12.757 \mathrm{~s}$ & $18.458 \mathrm{~s}$ & 293,250 & 2.8201 \\
$h / 2$ & $3.18925 \mathrm{~s}$ & $4.6145 \mathrm{~s}$ & 73,356 & 2.8934 \\
\hline
\end{tabular}

\section{Discussion}

In this research article, Crank Nicolson scheme has been successfully applied to find the solutions of two-dimension nonlinear reaction diffusion system. The accuracy and stability of the scheme demonstrated by test problem with data tables and figures. According to T Lakoba [32], implicit CN scheme is not efficient in term of computational time, as we see, the linear algebraic system from Jacobean matrix which is Penta block diagonal [32] [33] [34], with two block diagonals adjacent and two are unpaired at the distance of $L$ from main diagonal [33] [34] [35]. During simulation, unpaired block diagonals increase 


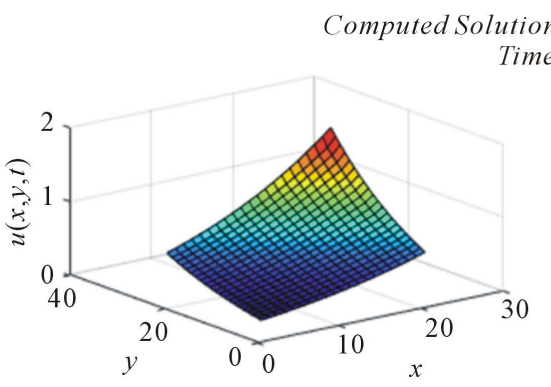

tion with Crank Nicolson

ime $t=0.1$
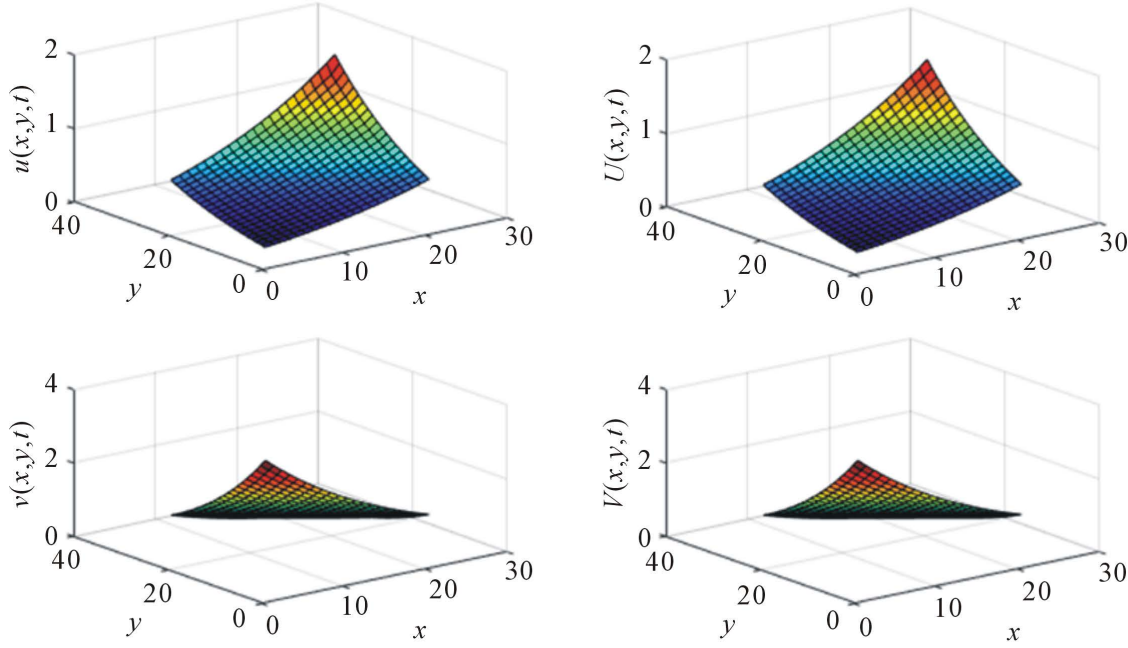

Figure 1. Shows results using Crank Nicolson scheme with $t=0.1$ and Grid $=25 \times 25$.

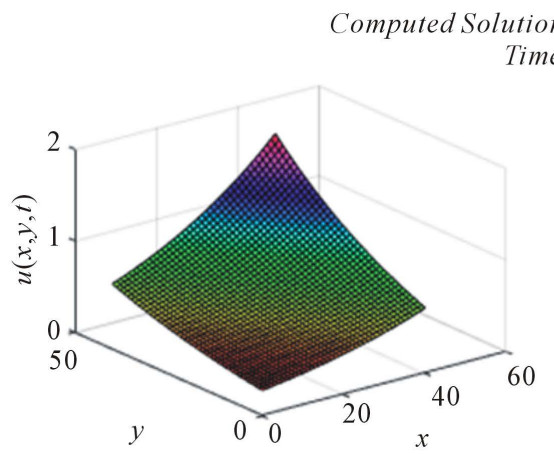

Time $t=0.3$
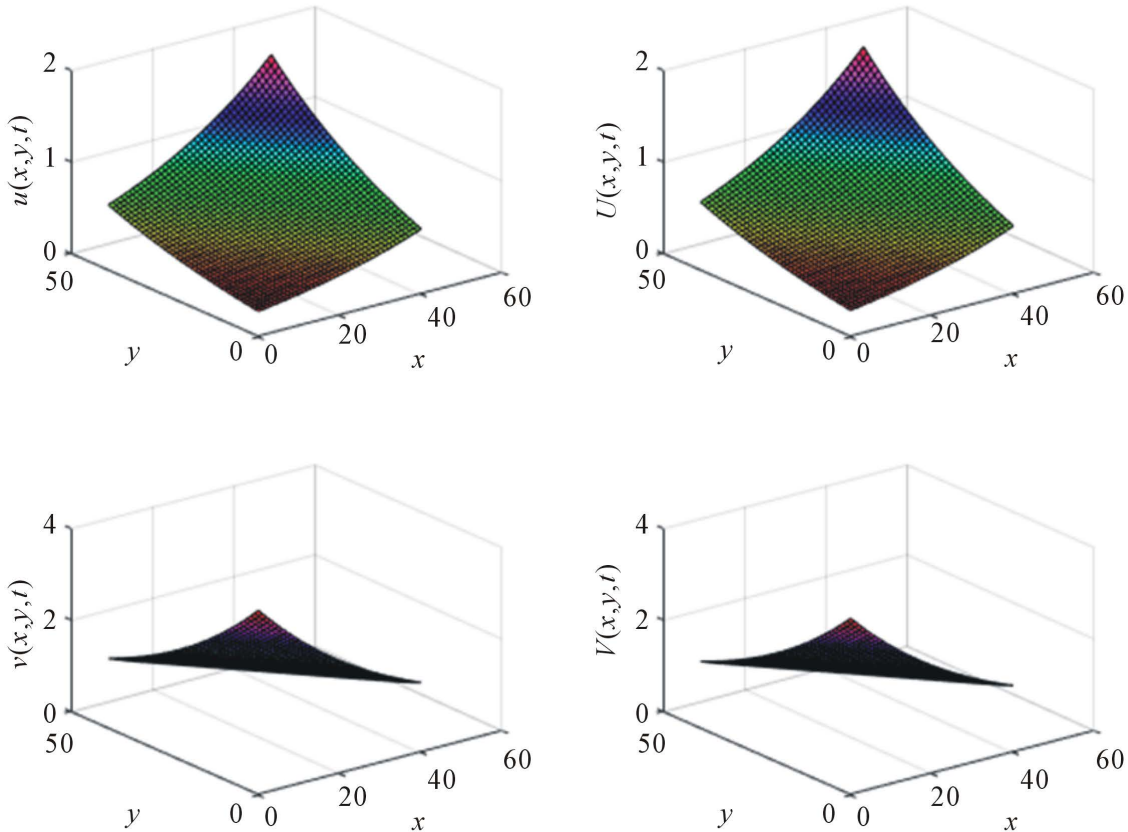

Figure 2. Shows results using Crank Nicolson scheme $t=0.3$ and Grid $=53 \times 53$.

computational time and increase memory capacity [34] [35] [36]. Solving such a linear system is not practical due to extremely high time complexity of solving a linear system by the means of Gaussian Elimination method or residual technique [34] [35] [36]. Hence an Alternating Direction Implicit (ADI) scheme can be implemented to solve the numerical PDE whereby one dimension is treated implicitly and other dimension explicitly for half of the assigned time step and vice versa for the remainder half of the time step [35] [36]. The benefit of this strategy 


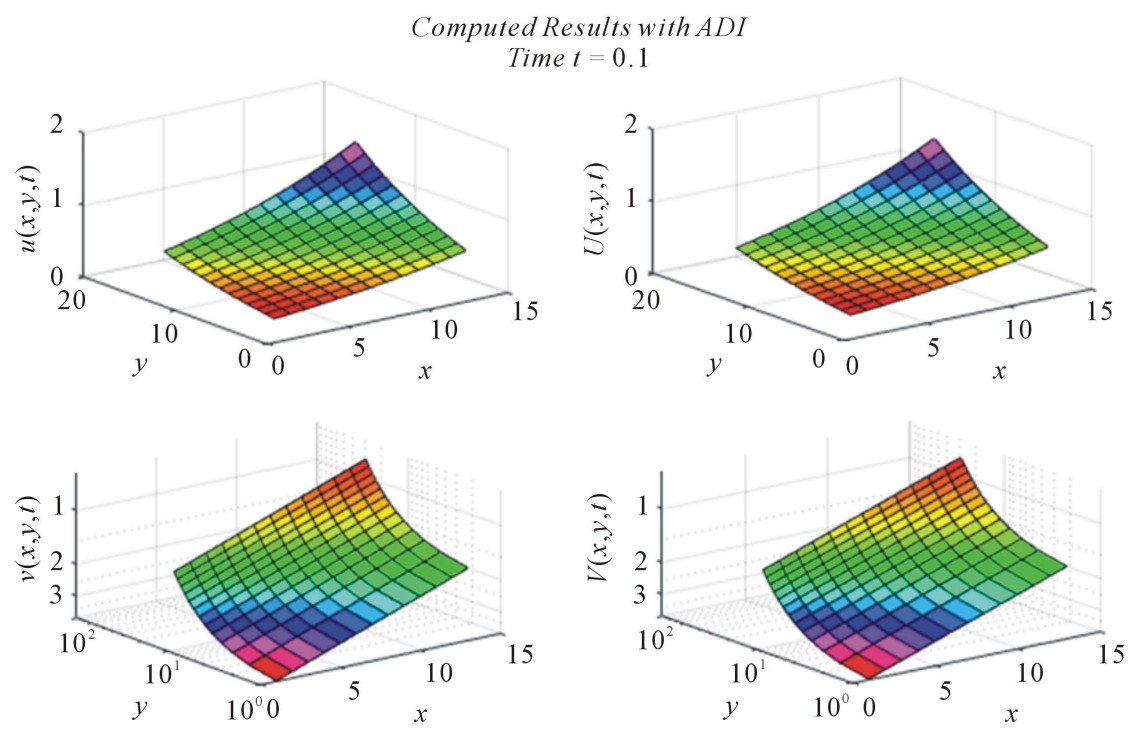

Figure 3. Shows results using ADI scheme with $t=0.1$ and Grid $=53 \times 53$.
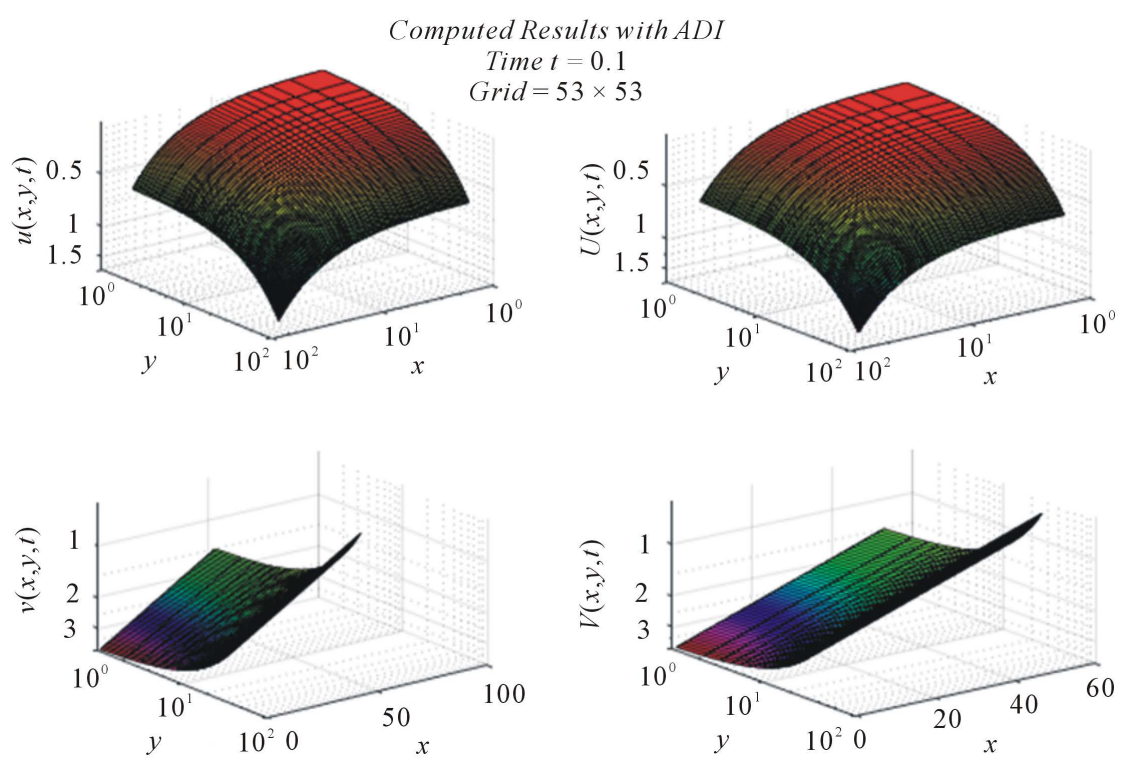

Figure 4. Shows results using ADI scheme with $t=0.1$ and Grid $=53 \times 53$ with $\log$ scaling in $x y$ plane of $u(x, y)$ and only $y \log$ scaling in $v(x, y)$.

is that the implicit solver only requires a tridiagonal matrix algorithm to be solved, so that the difference between the true Crank Nicolson solution and ADI approximated solution has an order of accuracy of $O\left(k^{2}\right)$ and hence can be ignored with a sufficiently small time-step [32]-[37]. The ADI method is a predictor corrector scheme where part of the difference operator is implicit in the initial (prediction) step and another part is implicit in the final (correction) step, whereas in ADI scheme two diagonals are paired with main diagonal and no other diagonals as in Crank Nicolson, thus these schemes reduce the computational time and increase efficiency [32]-[37]. The following diagrams indicate the 


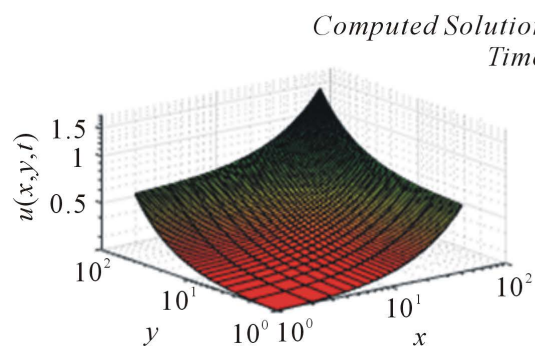

Time $t=0.5$
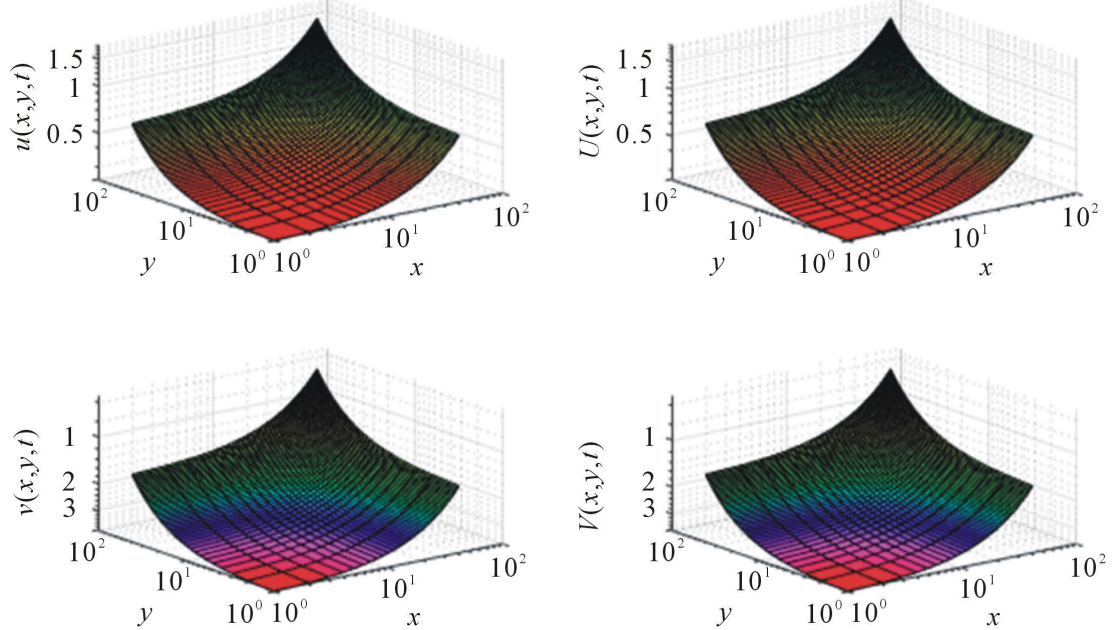

Figure 5. Shows results using $\mathrm{CN}$ scheme at $t=0.5$ and Grid $=101 \times 101$ with $\log$ scaling in $x y$ plane for $u(x, y)$ and $v(x, y)$ by Crank Nicolson.
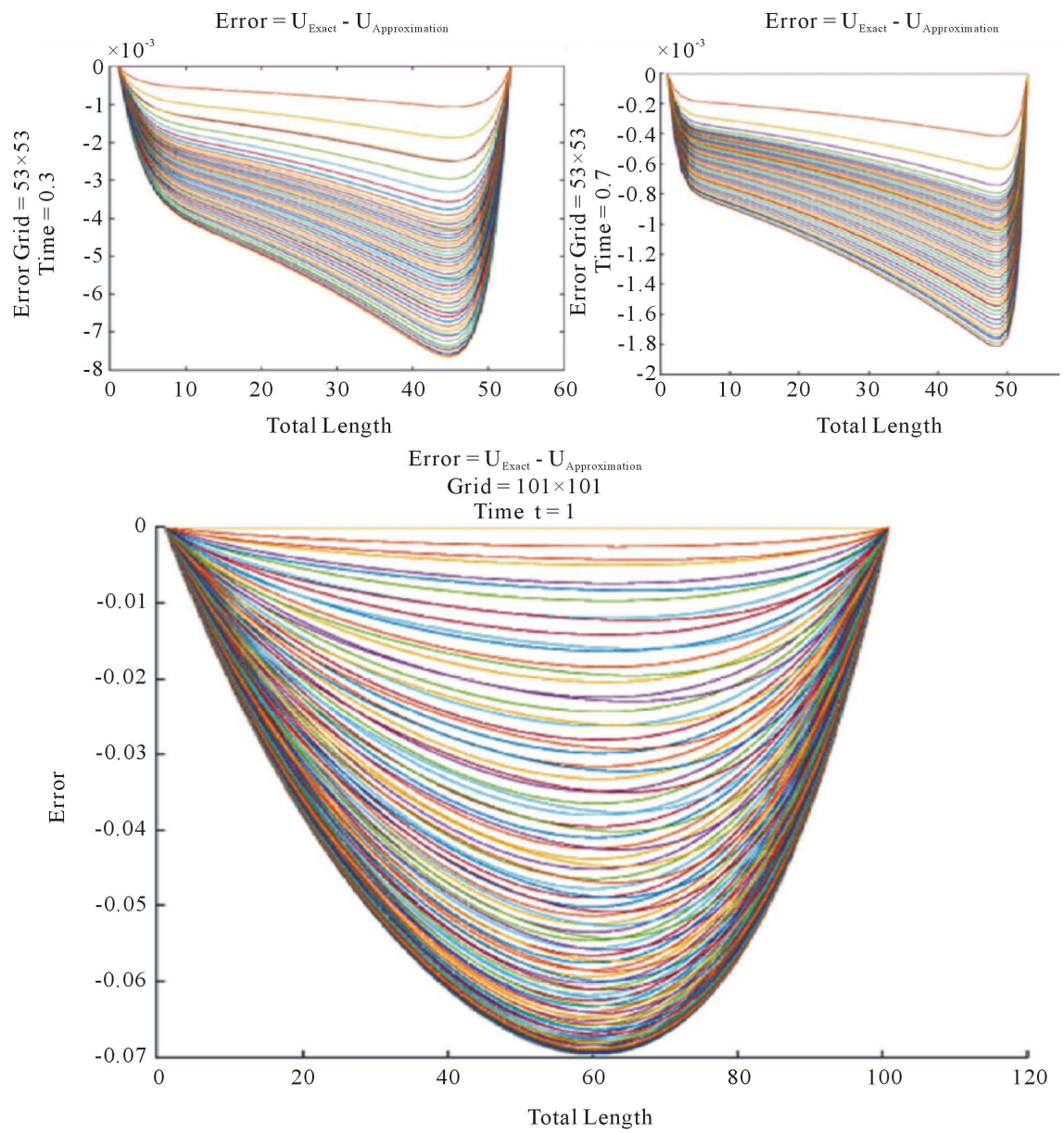

Figure 6. Shows results at different grids, for simple error in the concentration of the diffusion reaction system, with step up in grid sizes, make significant change in error but incremental in time, increase error as we see from Figure 6. 


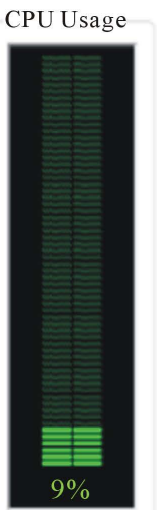

CPU Usage History
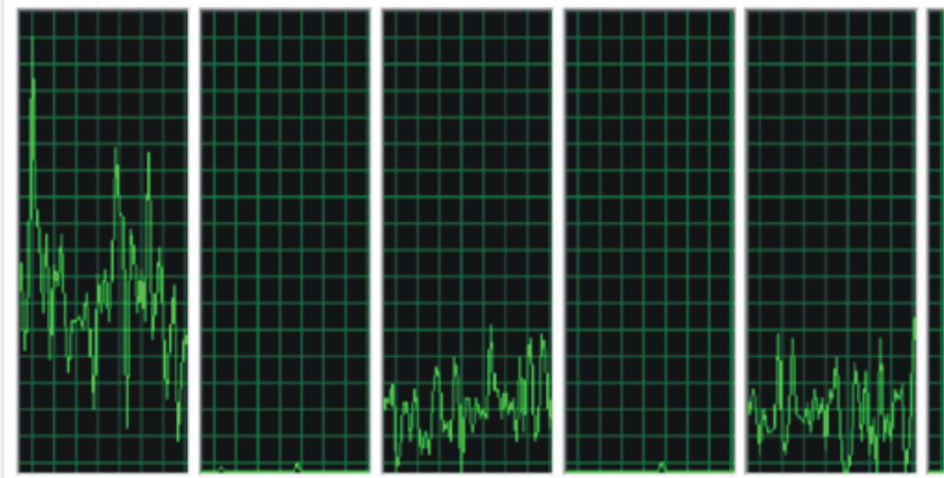

Memory

Physical Memory Usage History
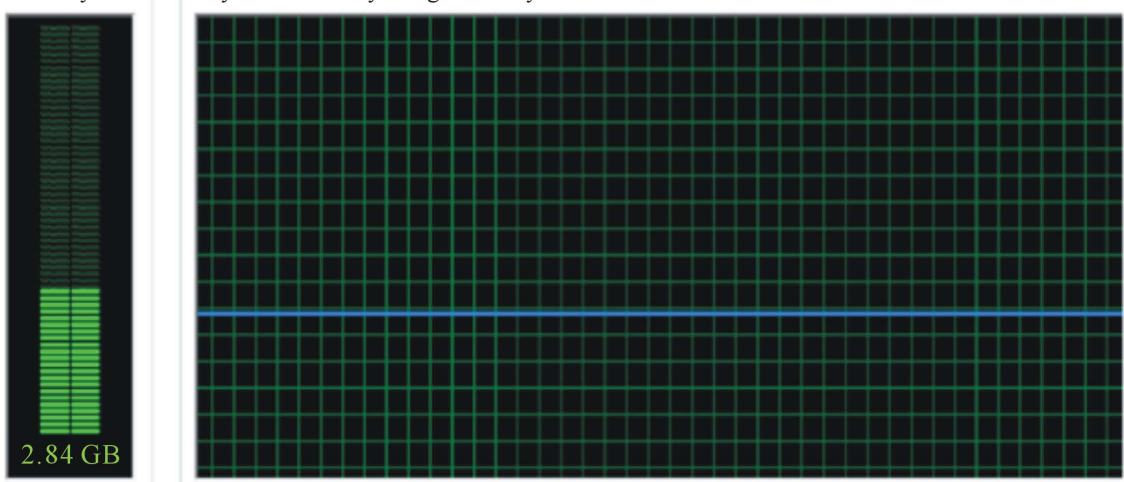

Physical Memory (MB)

Total

Cached

Available

Free

2943

System

Handles

Threads

Processes

Up Time

Commic (GB)

24875

1098

74

$0: 04: 24: 47$

Kernal Memory (MB)

Pages

2494

Nonpaged

336
180

4. Resource Monitor...

Figure 7. Shows simulations with capacity of the system along CPU usage and performance. Also processor calls and threads, by Crank Nicolson. Specification of the system is mentioned in computer applications header.

performance of the CPU for two different schemes. The derivation of our ADI scheme for a nonlinear PDE system relies on a few key observations. Most importantly, using the solution at time levels previous to $t=t^{n+1}$, the algorithm converts the nonlinear spatial operator into an implicit but linear operator with variable coefficients. The resulting approximately-factored equation is solved in sweeps along each of the Cartesian directions, including, as is common in ADI approaches, an intermediate $t^{n+1 / 2}$ step, so that all of the proposed algorithms are embodied in the two steps formula that every iteration updated the block tridiagonal linear algebraic system [32]-[39].

\section{Computer Applications}

Since last two decades, there is a challenging hasting among vendors and software development communities to bring improvement in performance for 

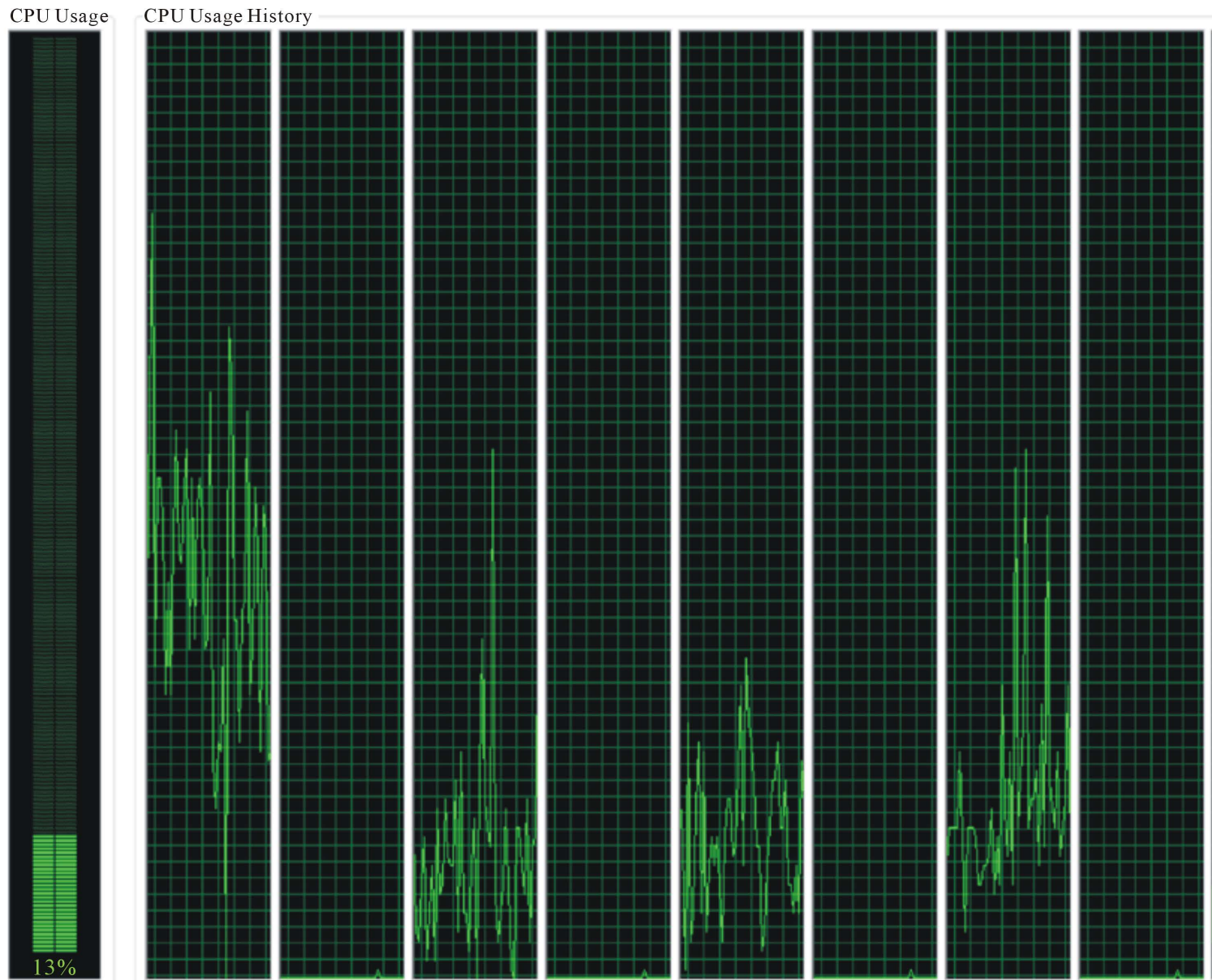

Figure 8. Shows simulations with ADI. CPU usage increase from $9 \%$ to $13 \%$ with efficiency.

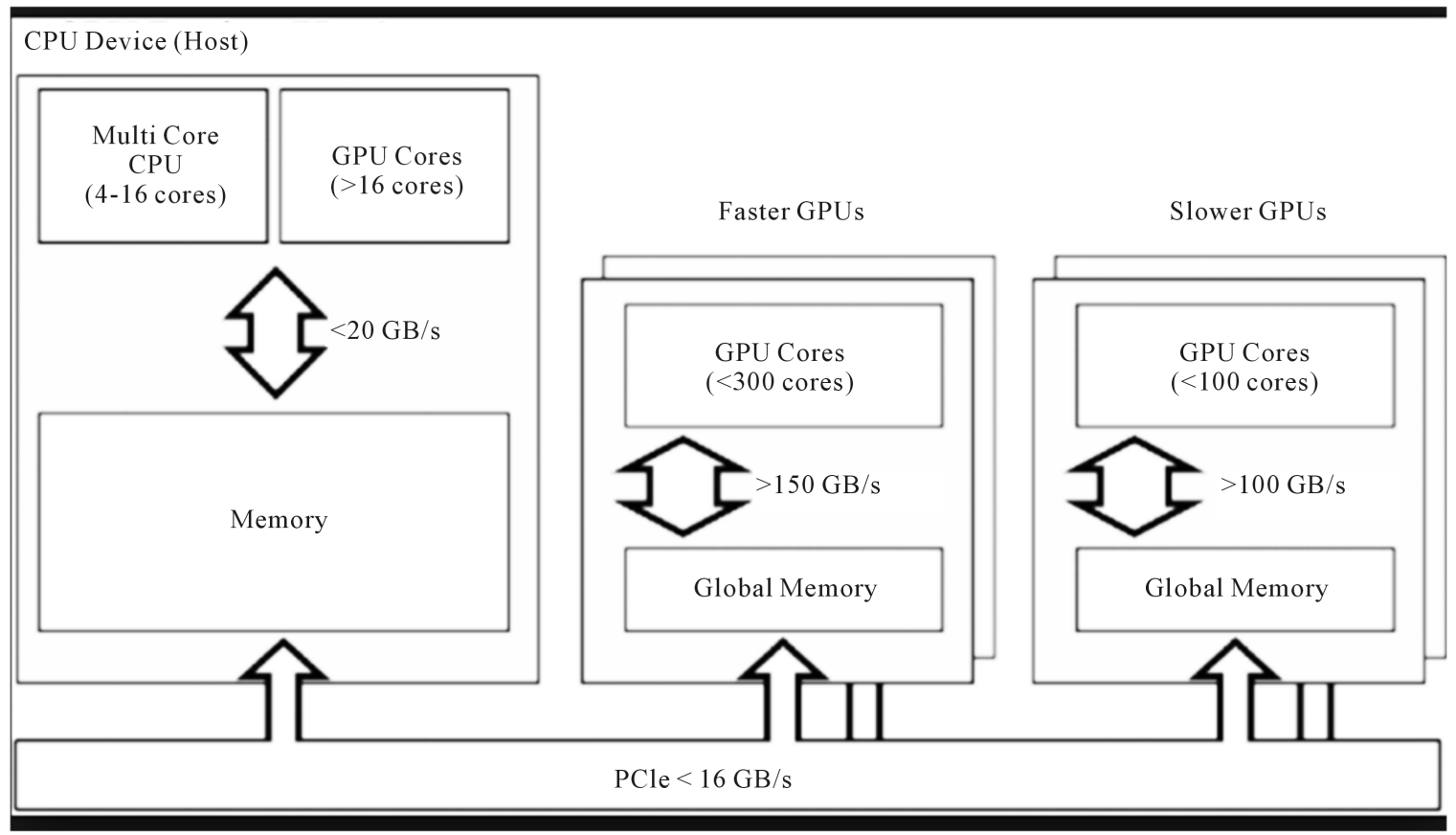

Figure 9. Shows GPU CPU collaboration. 
High performance computing system, by halting the traditional development to increase the clock rate, number of cores that are being increased in the system, however, many cores architecture based different powerful devices such GPU (Graphics Processing Unit) and GPGPU (General Purpose Graphics Processing Unit) by NIVIDA, MIC (Many Integrated Core) by Intel and FPGA (Field programmable Gate Array) have been introduced recently that outperform the conventional CPU processing by thousand folds [29] [38] [39]. In order to utilize these powerful devices, new software stacks, algorithms and frameworks have been introduced, ehereas the modern computing system provides massive parallelism through inter node and intra node computation where inter node processing is performed by MPI (Message Passing Interface), most popular programming model and intra node by OpenMP directive programing model [29] [38] [39].

Keeping in view, the advantages of this emerging technology, we have introduced Crank Nicolson and ADI schemes with the help of this application by using FUJITSU Primergy RX 350 S7 HPC computer having Intel Xeon E5-2667 processor of $2.80 \mathrm{GHz}$ processing power which contained 16 physical cores and 32 logical cores, main memory size of 32 GB and HDD 4 TB inside it [29] [38] [39], see from Figures 7-9. Moreover, we used 2 Tesla k-80 accelerated NVIDA devices that are capable to deliver not only graphical processing purpose but also for general purpose processing [29] [38] [39]. However, our application structure is designed for MATLAB based on hybrid of tri-hierarchy level tightly coupled programming model containing CUDA (Compute Uniform Device Architecture) for accelerated computation [29] [38] [39].

\section{Acknowledgements}

The authors are gratefully acknowledged Dr. Muhammad Faheem Afzaal, Department of Chemical Engineering, Imperial College London, UK and Muhammad Usman Ashraf, Department of Computer Science, King Abdulaziz University, Saudi Arabia.

\section{Conflict of Interest}

The authors do not have any conflict of interest in this research paper.

\section{References}

[1] Fisher, R.A. (1937) The Wave of Advance of Advantageous Genes. Annals of Eugenics, 7, 355-369. https://doi.org/10.1111/j.1469-1809.1937.tb02153.x

[2] Canosa, J.C. (1973) On a Nonlinear Diffusion Equation Describing Population Growth. IBM Journal of Research Development, 17, 307-313. https://doi.org/10.1147/rd.174.0307

[3] Arnold, A.R., Showalter, K.S. and Tyson, J.J. (1987) Propagation of Chemical Reactions in Space. Journal of Chemical Education, 64, 740-743. https://doi.org/10.1021/ed064p740

[4] Tuckwell, H.C. (1988) Introduction to Theoretical Neurobiology. Cambridge Uni- 
versity Press, Cambridge, UK.

[5] Gazdag, G.J. and Canosa, J.C. (1974) Numerical Solutions of Fisher's Equation. Journal of Applied Probability, 11, 445-457. https://doi.org/10.2307/3212689

[6] Abdullaev, U.G. (1994) Stability of Symmetric Traveling Waves in the Cauchy Problem for the KPP Equation. Journal of Differential Equations, 30, 377-386.

[7] Logan, D.J. (1994) An Introduction to Nonlinear Partial Differential Equations. Wiley, New York.

[8] Evans, D.J. and Sahimi, M.S. (1989) The Alternating Group Explicit (AGE) Iterative Method to Solve Parabolic and Hyperbolic Partial Differential Equations. Annals of Numerical Fluid Mechanics and Heat Transfer, 2, 283-389.

[9] Segel, L.A. (1984) Chapter 8: Modelling Dynamic Phenomena in Molecular and Cellular Biology. Cambridge University Press, Cambridge.

[10] Pao, C.V. (1981) Asymptotic Stability of Reaction-Diffusion Systems in Chemical Reactor and Combustion Theory. Journal of Mathematical Analysis and Applications, 82, 503-526. https://doi.org/10.1016/0022-247X(81)90213-4

[11] Schiesser, W.E. and Griffiths, G.W. (2009) A Compendium of Partial Differential Equation Models. Cambridge University Press, Cambridge. https://doi.org/10.1017/CBO9780511576270

[12] Argyris, J.A., Haase, M.H. and Heinrich, J.C. (1991) Finite Approximation to Two-Dimensional Sine Gordon Equations. Computer Methods in Applied Mechanics and Engineering, 86, 1-26. https://doi.org/10.1016/0045-7825(91)90136-T

[13] Aronson, D.G. and Weinberger, H.F. (1978) Multidimensional Non-Linear Diffusion Arising in Population Genetics. Advance in Mathematics, 30, 33-76.

https://doi.org/10.1016/0001-8708(78)90130-5

[14] Hilborn, R.H. (1975) The Effect of Spatial Heterogeneity on the Persistence of Predator-Prey Interactions. Theoretical Population Biology, 8, 346-355. https://doi.org/10.1016/0040-5809(75)90051-9

[15] Hans, L.P. (1999) Computational Partial Differential Equations. Springer Verlag, Berlin.

[16] Hayhoe, M.N. (1978) Numerical Study of Quasi-Analytic and Finite Difference Solutions of the Soil-Water Transfer Function. Soil Science, 125, 68-79.

[17] Linker, P.L. (1990) Numerical Methods for Solving the Reactive Diffusion Equation in Complex Geometries. Technische Universität Darmstadt, Darmstadt, Hessen.

[18] Tang, T.S. and Weber, R.O. (1991) Numerical Study of Fisher's Equations by a Petrov-Galerkin Finite Element Method. The ANZIAM Journal, 33, 27-38. https://doi.org/10.1017/S0334270000008602

[19] Khaled, K.A. (2001) Numerical Study of Fisher's Diffusion-Reaction Equation by the Sinc Collocation Method. Journal of Computational and Applied Mathematics, 137, 245-255. https://doi.org/10.1016/S0377-0427(01)00356-9

[20] Ames, W.F. (1965) Nonlinear Partial Differential Equations in Engineering. Academic Press, New York.

[21] Ames, W.F. (1969) Numerical Methods for Partial Differential Equations. Barnes and Noble, Inc., New York.

[22] Noye, J.N. (1989) Finite Difference Methods for Partial Differential Equations. North-Holland Publishing Comp. Conference in Queen's College, University of Melbourne, Australia, 23-87.

[23] Mittal, R.C. and Kumar, S.K. (2009) Numerical Study of Fisher's Equation by 
Wavelet Galerkin Method. International Journal of Computer Mathematics, 83, 287-298.

[24] Dhawam, S.D., Kapoor, S.K. and Kumar, S.K. (2012) Numerical Method for Advection Diffusion Equation Using FEM and B-Splines. Journal of Computer Science, 3, 429-437. https://doi.org/10.1016/j.jocs.2012.06.006

[25] Tamseer, M.T., Srivastava, V.K. and Mishra, P.D. (2016) Numerical Simulation of Three Dimensional Advection-Diffusion Equations by Using Modified Cubic B-Spline Differential Quadrature Method. Asia Pacific Journal of Engineering Science and Technology, 2, 1-13.

[26] Fletcher, C.A. (2016) Generating Exact Solutions of the Two-Dimensional Burgers Equations. International Journal for Numerical Methods in Fluids, 3, 213-216. https://doi.org/10.1002/fld.1650030302

[27] Hill, M.D. and Michael, R.M. (2008) Amdahl's Law in the Multicore Era. Computer, 41, 33-38. https://doi.org/10.1109/MC.2008.209

[28] Heath, M.T. (1997) Scientific Computing, an Introductory Survey. University of Illinois at Urbana-Champaign, Urbana and Champaign, IL.

[29] Ashraf, M.U., Fouz, F.F. and Fathy, A.E. (2016) Empirical Analysis of HPC Using Different Programming Models. International Journal of Modern Education and Computer Science, 6, 27-34.

[30] Busenberg, S.N. and Travis, C.C. (1983) Epidemic Models with Spatial Spread Due to Population Migration. Journal of Mathematical Biology, 16, 181-198. https://doi.org/10.1007/BF00276056

[31] Crank, J.C. and Nicolson, P.N. (1947) A Practical Method for the Numerical Evaluation of Solutions of Partial Differential Equations of the Heat-Conduction Type. Mathematical Proceedings of the Cambridge Philosophical Society, 43, 50-67. https://doi.org/10.1017/S0305004100023197

[32] Lakoba, T.L. (2015) The Heat Equation in 2 and 3 Spatial Dimensions. In: MATH 337, University of Vermont.

[33] Srivastava, V.K. and Tamsir, M.T. (2012) Crank-Nicolson Semi-Implicit Approach for Numerical Solutions of Two-Dimensional Coupled Nonlinear Burgers' Equations. International Journal of Applied Mechanics and Engineering, 17, 571-581.

[34] Smith, G.D. (1986) Numerical Solution of Partial Differential Equations: Finite Difference Methods. 3rd Edition, Oxford University Press, Oxford.

[35] Saber, E.N. (1999) An Introduction to Differential Equations. 2nd Edition, Springer Verlag, New York.

[36] Shalf, J.S., Sundip, D.S. and John, M.J. (2010) Exascale Computing Technology Challenges. In: Palma, J.M.L.M., Daydé, M., Marques, O. and Lopes, J.C., Eds., High Performance Computing for Computational Science-VECPAR 2010, Lecture Notes in Computer Science, Vol. 6449, Springer, Berlin, Heidelberg, 1-25.

[37] Shekhar, B.S. (2007) Thousand Core Chips: A Technology Perspective. Proceedings of the 44th annual Design Automation Conference, San Diego, CA, 4-8 June 2007, 746-749.

[38] Bajellan, A.A.A.F. (2015) Computation of the Convection-Diffusion Equation by the Fourth-Order Compact Finite Difference Method. İzmir Institute of Technology, January 2015.

[39] Ge, Y., Tian, Z.F. and Zhang, J. (2013) An Exponential HO Compact ADI Method for 3D Unsteady Convection Diffusion Problems. Numerical Methods for Partial Differential Equations, 29, 186-205. https://doi.org/10.1002/num.21705 